\title{
Insulin resistance and its metabolic, lipid and cardiovascular consequences
}

\begin{abstract}
Many research groups on atherosclerosis have sought through prospective large-scale epidemiological studies to better understand which residual factors would be associated with cardiovascular risk. Thus, atherogenic dyslipidemia was defined, such as the presence in an individual of decreased HDL-C levels, increased triglyceride levels, and a relatively high proportion of small and dense LDL-C particles. On the other hand, it was found that atherogenic dyslipidemia is present in cases of insulin resistance and metabolic syndrome (low HDL-C and elevated triglycerides are part of the definition of this syndrome) and consequently in patients with type 2 diabetes mellitus. Regarding treatment, there are studies in diabetic patients with risk reduction with fenofibrate, and the guidelines recommend the association of fenofibrate with statins. Diabetes mellitus is also an important cause of hospitalizations and proportional mortality, also assuming that most deaths register only the immediate cause of this death, which is often the result of diabetes complications. Most of these complications are cardiovascular diseases, which may manifest as coronary heart disease, cerebrovascular disease or peripheral arteriopathies. These are the so-called macrovascular complications of type 2 diabetes and are present even before the onset of hyperglycemia, due to the presence of insulin resistance and associated metabolic syndrome. Metabolic syndrome is characterized by the presence in the patient of at least 3 out of 5 parameters (increased abdominal waist, high glycemia, hypertriglyceridemia, low HDL-C and arterial hypertension) and is one of the factors responsible for the macrovascular changes.
\end{abstract}

Keywords: apolipoprotein B, atherogenic dyslipidemia, HDL-C, LDL-C, plasminogen activator inhibitor Type 1, peroxisome proliferator activator activated receptors, VLDL
Volume I4 Issue 3 - 202I

Sidney Carvalho Fernandes, 'Anita L. R. Saldanha,' Ana Paula Pantoja Margeotto,' André Luis Valera Gasparoto, ${ }^{2}$ José Mendes Aldrighi,' Marco Antonio De Vivo Barros,' Tania Leme da Rocha Martinez'

'Department of Nephrology,A Beneficência Portuguesa de São Paulo, Brazil

${ }^{2}$ Intensive Care Unit,A Beneficência Portuguesa de São Paulo, Brazil

Correspondence: Tania Leme da Rocha Martinez, BP - A Beneficência Portuguesa de São Paulo, Rua Comandante Ismael Guilherme, 358 - Jardim Lusitânia, CEP 0403।- 120 - São Paulo SP, Brazil,Tel 55 || 98323-9863, Fax 55 || 3842-3789, Email tamar@uol.com.br

Received: February 28, 2021 | Published: May 03, 2021
Abbreviations: AD, Atherogenic dyslipidemia; Apo B, Apolipoprotein Bl; HDL-C, High Density Lipoprotein Cholesterol; LDL-C, Low Density Lipoprotein Cholesterol; PAI-1, Plasminogen Activator Inhibitor Type 1; PPARs, Peroxisome Proliferator Activator Activated Receptors; VLDL, Very Low Density Lipoprotein; VLDL-C, Very Low Density Lipoprotein Cholesterol

\section{Mini review}

It has been known for a long time that about $35 \%$ of cases of coronary artery disease occur in individuals with total cholesterol below $200 \mathrm{mg} / \mathrm{dL}){ }^{1}$

Considering that the average total cholesterol in populations of developed and developing countries ranges between 200 and 220mg/ $\mathrm{dL}$ we have at least half of the cases of coronary artery disease and an even higher percentage of strokes will occur in individuals with below average total cholesterol levels. For this reason, many research groups on atherosclerosis have sought through prospective largescale epidemiological studies to better understand what other factors would be associated with cardiovascular risk. It was then found that patients with low High Density Lipoprotein Cholesterol (HDL-C), high triglycerides and with a high percentage of small and dense Low Density Lipoprotein Cholesterol (LDL-C) particles had a high cardiovascular risk in relation to individuals not with these alterations.

Atherogenic dyslipidemia (AD) was thus defined as the presence in an individual of decreased HDL-C levels, increased triglyceride levels, and a relatively high proportion of small and dense LDL-C particles.
On the other hand, it was found that $\mathrm{AD}$ is present in cases of insulin resistance and metabolic syndrome (low HDL-C and elevated triglycerides are part of the definition of this syndrome) and consequently in patients with type 2 diabetes mellitus. Although $\mathrm{AD}$ can also be called diabetic dyslipidemia, it should be emphasized that it is also present in the absence of it and even in patients who do not present metabolic syndrome.

We already know today the etiophysiological changes associated with AD: in patients with hypertriglyceridemia (triglycerides above $150 \mathrm{mg} / \mathrm{dL}$ ) an excess of fatty acids occurs in the liver, with increased apolipoprotein B (apoB) by decreasing its degradation with consequent increase in the synthesis of Very Low Density Lipoprotein (VLDL) rich in triglycerides. For any LDL level there is an increase in cases of coronary disease even moderate hypertriglyceridemia. ${ }^{2} \mathrm{We}$ also add that hypertriglyceridemia is more common in early coronary disease than hypercholesterolemia. ${ }^{3}$

This excess of VLDL, suffering actions of various enzymes, ends up leading to the formation of small and dense LDLs and a decrease in HDL concentration. These small, dense LDLs are more atherogenic due to two factors: easier to cross the endothelium and greater tendency to oxidation. The presence of these LDL can still mask a high number of LDL particles.

There is another factor to be considered is the increase in apoB, which, as demonstrated in the INTERHEART ${ }^{4}$ and AMORIS ${ }^{5}$ studies, is a better risk indicator than LDL.

It is also known that $\mathrm{AD}$ is associated with high fibrinogen levels and Plasminogen Activator Inhibitor (PAI-1) ${ }^{6}$ thus presenting these 
patients, with a higher risk of atherothrombotic phenomena. Diabetes mellitus is currently a disease of great prevalence worldwide and in Brazil it is among the highest in the adult population. In a study conducted in our country, it was found that about $46 \%$ of the diagnosed patients did not know how to have this condition. ${ }^{7}$

Diabetes mellitus is also an important cause of hospitalizations and proportional mortality, also assuming that most deaths register only the immediate cause of this death, which is often the result of diabetes complications. Most of these complications are cardiovascular diseases, which may manifest as coronary heart disease, cerebrovascular disease or peripheral arteriopathies. These are the so-called macrovascular complications of type 2 diabetes and are present even before the onset of hyperglycemia, due to the presence of insulin resistance and associated metabolic syndrome. Metabolic syndrome is characterized by the presence in the patient of at least 3 out of 5 parameters (increased abdominal waist, high glycemia, hypertriglyceridemia, low HDL-C and arterial hypertension) and is one of the factors responsible for the macrovascular changes mentioned above. Due to this factor, patients with type 2 diabetes need a stricter control of their lipid level, blood pressure and lifestyle, avoiding smoking, sedentary lifestyle and an inadequate diet. However, even after correcting these factors, the patient with type 2 diabetes persists with an excess of cardiovascular complications when compared to a person without diabetes. The CARDS study shows that the association of atorvastatin with diabetic patients reduced the incidence of acute coronary disease by $36 \%$, myocardial revascularization by $31 \%$, stroke by $48 \%$ and overall mortality by $27 \%(\mathrm{p}=0.059)^{8}$ but still an excess of events in treated patients.

We then have that, the patient with type 2 diabetes, even with their risk factors kept under control presents an excess of vascular morbidity and mortality, due to a factor that was called residual cardiovascular risk. We know today that the hidden enemy responsible for this residual risk is $\mathrm{AD}$.

The most common lipid disorder in diabetic patients is hypertriglyceridemia caused by an increase in very low density lipoprotein cholesterol (VLDL-C) particles around 50 to $100 \%$. Diabetic patients with very high triglyceride level $(>400 \mathrm{mg} / \mathrm{dL})$ should also have genetic defects in the metabolism of lipoproteins independent of diabetes.

We therefore know that only the use of statins, even the most potent ones, cannot reduce cardiovascular risk in patients with $\mathrm{AD}$ whether or not these patients have diabetes mellitus or metabolic syndrome.

Certain nuclear receptors, known as Peroxisome Proliferator Activator Receptors (PPARs) have the ability to modulate various metabolic steps related to the atherogenesis process. These receptors are activated by endogenous ligands (some lipids e.g.) or exogenous (the class of drugs generically referred to as fibrates). The final effect of binding fibrates to these nuclear receptors is an increase in HDL particles and a decrease in triglycerides and a decrease in small and dense LDL particles. We therefore see that PPAR activators act by modulating the main factors involved in the onset of atherogenesis. Statins and fibrates act synergistically throughout the atherothrombosis process.

What strategy should we then adopt to reduce residual cardiovascular risk in patients with $\mathrm{AD}$, who already receive statins and maintain an adequate level of LDL-C? In a recent consensus of recently published European experts, Ferrari et al. review the use of statins (and if necessary ezetimibe) with fenofibrate ${ }^{9}$, based mainly on the ACCORD study. ${ }^{10}$ This strategy was also recognized as valid by the Spanish Society of Arteriosclerosis, which began to recommend the association of fenofibrate with statins with or without ezetimib to reduce residual cardiovascular risk. ${ }^{11}$

Today we consider the introduction of mandatory phenofibrate in patients with a high triglyceride level $(400 \mathrm{mg} / \mathrm{dL})$ and also in cases of AD, especially if triglyceride levels are greater than $200 \mathrm{mg} / \mathrm{dL} .{ }^{12}$

In a recent study in Japanese diabetic patients comparing the effect of statins (syvastatin, atorvastatin and rosvastatin) versus a combination of fenofibrate with ezetimibe, Shinnakasu et al. demonstrated that this combination of lipid-lowering agents was significantly better than statin alone in decreasing triglycerides, VLDL, triglycerides linked to chylomicrons, triglycerides bound to HDL, oxidized LDL and also in the increase of HDL. There was an increase in LDL diameter and decrease in HDL diameter (also at a statistically significant level). The vascular function measured by flow-mediated dilation also improved significantly with the use of association, which was attributed to the increase of the smaller particles of HDL. ${ }^{13}$

With the objective of reducing the number of tablets used daily, a study was also carried out with the association of pravastatin with fenofibrate in a fixed-dose combination (40mg pravastatin and $160 \mathrm{mg}$ fenofibrate), which was shown to be well tolerated. ${ }^{14}$

\section{Conclusion}

In relation to the association of statins with other fibrates there are no studies showing their efficacy or safety, hence the exclusive use of fenofibrate in this indication.

\section{Acknowledgments}

None.

\section{Conflicts of interest}

Author declares that there are no conflicts of interest.

\section{Funding}

None.

\section{References}

1. Castelli WP. Lipids, risk factors and ischaemic heart disease Atherosclerosis. 1996;124(Supp1):S1-S9.

2. Martin-Nizard F, Furman C, Delerive P, et al. Peroxisome proliferatoractivated receptor activators inhibit oxidized low-density lipoproteininduced endothelin-1 secretion in endothelial cells. $J$ Cardiovasc Pharmacol. 2002;40(6):822-831.

3. Rubins HB, Robins SJ, Collins D, et al. Distribution of lipids in 8,500 men with coronary artery disease. Department of Veterans Affairs HDL Intervention Trial Study Group. Am J Cardiol. 1995;75(17):1196-1201.

4. Yusuf S, Hawken S, Ounpuu S, et al. Effect of potentially modifiable risk factors associated with myocardial infarction in 52 countries (the INTERHEART study):case-control study. Lancet. 2004;364(9438):937952.

5. McQueen MJ, Hawken S, Wang X, et al. Lipids, lipoproteins, and apolipoproteins as risk markers of myocardial infarction in 52 countries (the INTERHEART study):a case-control study. Lancet. 2008;372(9634):224-233.

6. Okopien B, Cwalina L, Lebek M, et al. Effects of fibrates on plasma prothrombotic activity in patients with type IIb dyslipidemia. Int $J$ Clin Pharmacol Ther. 2001;39(12):551-557. 
7. Malerbi DA, Franco LJ. Multicenter study of the prevalence of diabetes mellitus and impaired glucose tolerance in the urban Brazilian population aged 30-69 yr. The Brazilian Cooperative Group on the Study of Diabetes Prevalence. Diabetes Care. 1992;15(11):1509-1516.

8. Colhoun HM, Betteridge DJ, Durrington PN, et al. Primary prevention of cardiovascular disease with atorvastatin in type 2 diabetes in the Collaborative Atorvastatin Diabetes Study (CARDS):multicentre randomised placebo-controlled trial. Lancet. 2004;364(9435):685-696.

9. Ferrari R, Aguiar C, Alegria E, et al. Current practice in identifying and treating cardiovascular risk, with a focus on residual risk associated with atherogenic dyslipidaemia. Eur Heart J Suppl. 2016;18(Suppl C):C2-C12.

10. ACCORD Study Group, Ginsberg HN, Elam MB, et al. Effects of combination lipid therapy in type 2 diabetes mellitus. $N$ Engl $\mathrm{J} \mathrm{Med}$. 2010;362(17):1563-1574.

11. Grupo de trabajo de Dislipemia Aterogénica de la Sociedad Española de Arteriosclerosis y Grupo Europeo de Expertos. Practical recommendations for the management of cardiovascular risk associated with atherogenic dyslipidemia, with special attention to residual risk. Spanish adaptation of a European Consensus of Experts. Clin Investig Arterioscler. 2017;29(4):168-177.
12. Tenenbaum A, Fisman EZ. Fibrates are an essential part of modern antidyslipidemic arsenal:spotlight on atherogenic dyslipidemia and residual risk reduction. Cardiovasc Diabetol. 2012;11:125.

13. Shinnakasu A, Yamamoto K, Kurano M, et al. The Combination Therapy of Fenofibrate and Ezetimibe Improved Lipid Profile and Vascular Function Compared with Statins in Patients with Type 2 Diabetes. J Atheroscler Thromb. 2017;24(7):735-748.

14. Hernández Mijares A. Combination of pravastatin and fenofibrate (Pravafenix ${ }^{\circledR}$ ). Safety studies. Clin Investig Arterioscler. 2014;26(Suppl 1):25-30. 